\title{
Holomorphic Parabolic Geometries and Calabi-Yau Manifolds
}

Benjamin MCKAY

School of Mathematical Sciences, University College Cork, Cork, Ireland

E-mail: b.mckay@ucc.ie

URL: http://euclid.ucc.ie/pages/staff/Mckay/

Received May 25, 2011, in final form September 15, 2011; Published online September 20, 2011

http://dx.doi.org/10.3842/SIGMA.2011.090

\begin{abstract}
We prove that the only complex parabolic geometries on Calabi-Yau manifolds are the homogeneous geometries on complex tori. We also classify the complex parabolic geometries on homogeneous compact Kähler manifolds.
\end{abstract}

Key words: parabolic geometry; Calabi-Yau manifold

2010 Mathematics Subject Classification: 53C55; 53A55; 53C10

\section{Introduction}

We will prove that Calabi-Yau manifolds (other than those covered by complex tori) cannot bear holomorphic parabolic geometries. Gunning [12] proved that any compact Kähler surface with $c_{1}=0$ admitting a holomorphic normal projective or conformal connection is covered by a complex torus. Kobayashi [21] proved that any compact Kähler manifold with $c_{1}=0$ which admits a holomorphic normal projective or conformal connection is covered by a complex torus. Our arguments are simpler than those of Gunning [12] or Kobayashi [21], and give stronger conclusions (not requiring normalcy, and applying directly to all holomorphic parabolic geometries, not just projective and conformal connections).

\section{Review of the literature}

Let us contrast our results in this paper with those of [3, 5, 10]. In [3], we proved that if a smooth complex projective variety with $c_{1}=0$ bears a holomorphic Cartan geometry, then the smooth projective variety has holomorphic unramified covering map by an Abelian variety. In [5], we generalized this result to prove that if a compact Kähler manifold with $c_{1}=0$ bears a holomorphic Cartan geometry, then the compact Kähler manifold has a holomorphic unramified covering map by a complex torus. A special case of this result (for parabolic geometries) will be proven here in the first part of Theorem 1. Sorin Dumitrescu proved this same result, and a collection of remarkable generalizations (for example obstructions to holomorphic Cartan geometries on products $C Y \times X)$. However, those proofs used algebraic geometry where the proof below relies more on the local theory of parabolic geometries. Dumitrescu also proved similar results to those below on Calabi-Yau manifolds bearing structures of affine algebraic type [8]. The paper you are currently reading improves in one way on all of those results: Theorem 3 shows that holomorphic parabolic geometries on tori are translation invariant. Moreover the proof of Theorem 3 uses exactly the same local calculations as the proof of Theorem 1. It is well known that there are holomorphic Cartan geometries on complex tori which are not translation invariant, so Theorem 3 is surprising. The classification of holomorphic parabolic geometries on compact Kähler manifolds with $c_{1}=0$ is completed below (up to finite unramified covering), in 
Theorems 1 and 3. This classification is then use to classify holomorphic parabolic geometries on homogeneous compact Kähler manifolds. The analogous classifications for holomorphic Cartan geometries are not known or conjectured.

Let us review the current state of the search for holomorphic Cartan geometries on compact complex manifolds. Holomorphic Cartan geometries are completely classified on (1) any compact Riemann surface [27], (2) any compact complex surface containing a rational curve [27] and (3) any compact rationally connected complex manifold [4]. (This last class of manifolds includes, for example, all Fano manifolds, and all rational homogeneous varieties $G / P$.) On compact Kähler manifolds with $c_{1}=0$, we classify below all of the holomorphic parabolic geometries. Suppose that $P \subset G$ is a maximal parabolic subgroup of a complex semisimple Lie group. For any compact Kähler manifold $M$ containing a rational curve, either $M=G / P$ or else $M$ admits no holomorphic $G / P$-geometry [4].

On every compact complex surface, all holomorphic torsion-free affine connections are classified $[18,9]$. On every compact complex surface, all holomorphic normal projective connections which do not arise from holomorphic affine connections are classified [18, 9]. (It remains to see which pairs of holomorphic affine connections determine the same holomorphic projective connection.) It is known which compact complex surfaces admit holomorphic normal parabolic geometries [15, 21, 22, 23, 25].

Suppose that $M$ is a locally symmetric complex manifold of finite volume, $M=\Gamma \backslash X$, where $X$ is a noncompact Hermitian symmetric space, say with compact dual $G / P$, and $\Gamma$ is a discrete group of isomorphisms of the standard flat $G / P$-geometry on $X$. Suppose further that $G / P$ is an irreducible symmetric space. Then $M$ admits a unique normal holomorphic parabolic geometry modelled on $G / P$ (the obvious one) [24, 19].

On any compact Kähler manifold, there are constraints on characteristic classes arising from the presence of holomorphic Cartan geometries [24, 26]. The smooth complex projective 3 -folds that bear holomorphic normal projective or conformal connections are classified $[16,17]$.

Every known example of a compact complex manifold admitting a holomorphic Cartan geometry also admits a flat holomorphic Cartan geometry with the same model with one exception: there are translation invariant Cartan geometries on the complex torus (see below), which are not flat, and for which no complex torus admits a flat geometry with the same model. Many compact complex manifolds only admit locally homogeneous holomorphic Cartan geometries, but some also admit locally inhomogeneous ones [9]. There are sporadic results classifying flat holomorphic Cartan geometries of various types on various complex manifolds [28], but I am not aware of any other results concerning the classification of holomorphic Cartan geometries.

\section{Calabi-Yau manifolds}

Definition 1. For this article, a Calabi-Yau manifold is a compact Kähler manifold $M$ with $c_{1}(T M)=0$.

It is well known that a Calabi-Yau manifold satisfies $c_{2}(T M)=0$ just if it has a torus as unramified covering space. Let us recall how this follows from Yau's proof of the Calabi conjecture. For any Kähler manifold, say of dimension $n$, with $\Omega$ its Kähler form, it is easy to calculate that

$$
c_{2} \wedge \Omega^{n-2}=\left(\|R\|^{2}+\text { scalar }^{2}-2\|\operatorname{Ricci}\|^{2}\right) \Omega^{n},
$$

(see Berger and Lascoux [2]) where $R$ is the curvature tensor. If $c_{1}=0$, then there is a metric for which Ricci $=0$, by Yau's solution of the Calabi conjecture [34]. Hence $c_{2}=0$ implies $R=0$, flat. But then $M$ is covered by a flat torus (see Igusa [14]). 
Lemma 1 (Inoue, Kobayashi and Ochiai [15]). Any compact complex manifold which bears a holomorphic Cartan geometry with reductive algebraic structure group has vanishing Atiyah class. In particular, if Kähler then it is the quotient of a complex torus under a finite unramified covering map.

Proof. The Cartan connection splits invariantly into a sum of a connection (in the sense of Ehresmann) and a soldering form; see Sharpe [32, Lemma 2.1, p. 362]. The existence of a connection is precisely the vanishing of the Atiyah class; see Atiyah [1]. If Kähler, then all Chern classes of the tangent bundle vanish just when the Atiyah class does. By the previous discussion, the manifold has a torus as finite unramified covering space.

Example 1. A holomorphic Riemannian metric is a simple example of a reductive Cartan geometry, and our results tell us that holomorphic Riemannian metrics can not live on any compact Kähler manifold except those covered by tori. This is well known (see Inoue, Kobayashi and Ochiai [15]).

We will prove the following theorem.

Theorem 1. If a Calabi-Yau manifold bears a holomorphic parabolic geometry, then it is covered by a torus. More generally, any compact complex manifold with a holomorphic parabolic geometry and trivial canonical bundle must have a holomorphic affine connection.

\section{Rational homogeneous varieties}

Suppose that $G / P$ is a rational homogeneous variety, so $G$ is a complex semisimple Lie group and $P$ is a complex parabolic subgroup, with Lie algebras $\mathfrak{g}$ and $\mathfrak{p}$. We can express $\mathfrak{p}$ as a sum of the Cartan subalgebra of $\mathfrak{g}$ together with various root spaces, including all of the positive root spaces. Some negative root spaces will also lie in $\mathfrak{p}$. Once we fix the choice of $\mathfrak{g}$ and $\mathfrak{p}$, roots then divide up into 3 categories as follows. The compact roots of $\mathfrak{g}$ are the roots $\alpha$ of $\mathfrak{g}$ so that the root spaces of both $\alpha$ and $-\alpha$ belong to the Lie algebra of $\mathfrak{p}$. All other roots are noncompact, and divide into the noncompact positive and noncompact negative roots, according to whether or not their root spaces lie in $\mathfrak{p}$. The Dynkin diagram of $G / P$ is the Dynkin diagram of $G$ (labelled by simple roots), with simple roots dotted if they are compact, and crossed if they are noncompact.

The sum of the root spaces of the noncompact positive roots is the maximal nilpotent subalgebra, denoted $\mathfrak{n} \subset \mathfrak{p}$. The sum of the root spaces of the noncompact negative roots is also a nilpotent subalgebra, denoted $\mathfrak{n}^{-} \subset \mathfrak{g}$, complementary to $\mathfrak{p}$. Let $\mathfrak{a} \subset \mathfrak{p}$ be the subalgebra spanned by the coroots of the compact roots. Let $\mathfrak{m} \subset \mathfrak{p}$ be the Lie subalgebra generated by the root space of the compact roots. The Lie subalgebra $\mathfrak{m} \oplus \mathfrak{a}$ (n resp.) is the maximal reductive (nilpotent) subalgebra of $P$; see Knapp [20]. Let $M, A, N$ and $N^{-}$be the connected subgroups of $G$ with Lie algebras $\mathfrak{m}, \mathfrak{a}, \mathfrak{n}$ and $\mathfrak{n}^{-}$. The groups $M, A, N, N^{-}$and $P$ are all algebraic (see Fulton and Harris [11, p. 382]). The splitting $\mathfrak{g}=\mathfrak{n}^{-} \oplus \mathfrak{m} \oplus \mathfrak{a} \oplus \mathfrak{n}$ is $M A$-invariant.

Pick a Chevalley basis $X_{\alpha}, H_{\alpha}$ for $\mathfrak{g}$. Recall (see Serre [31]) that this is a basis parameterized by roots $\alpha \in \mathfrak{h}^{*}$ (with $\mathfrak{h} \subset \mathfrak{g}$ a Cartan subalgebra) for which

1. $\left[H, X_{\alpha}\right]=\alpha(H) X_{\alpha}$ for each $H \in \mathfrak{h}$;

2. $\alpha\left(H_{\beta}\right)=2 \frac{\langle\alpha, \beta\rangle}{\langle\beta, \beta\rangle}$ (measuring inner products via the Killing form);

3. $\left[H_{\alpha}, H_{\beta}\right]=0$;

4 .

$$
\left[X_{\alpha}, X_{\beta}\right]= \begin{cases}H_{\alpha}, & \text { if } \alpha+\beta=0 \\ N_{\alpha \beta} X_{\alpha+\beta}, & \text { otherwise }\end{cases}
$$


with

(a) $N_{\alpha \beta}$ an integer,

(b) $N_{-\alpha,-\beta}=-N_{\alpha \beta}$,

(c) If $\alpha, \beta$, and $\alpha+\beta$ are roots, then $N_{\alpha \beta}= \pm(p+1)$, where $p$ is the largest integer for which $\beta-p \alpha$ is a root,

(d) $N_{\alpha \beta}=0$ if $\alpha+\beta=0$ or if any of $\alpha, \beta$, or $\alpha+\beta$ is not a root.

Consider the 1-forms $\omega^{\alpha}$ dual to the vectors $X_{\alpha}$ of a Chevalley basis. We use the Killing form to extend $\alpha$ from $\mathfrak{h}$ to $\mathfrak{g}$, by splitting $\mathfrak{g}=\mathfrak{h}+\mathfrak{h}^{\perp}$, and taking $\alpha=0$ on $\mathfrak{h}^{\perp}$. The 1 -forms $\omega^{\alpha}, \alpha$ $\operatorname{span} \mathfrak{g}^{*}$.

Each exterior form in $\Lambda^{*}(\mathfrak{g})^{*}$ extends uniquely to a left invariant differential form in $\Omega^{*}(G)$, and we will identify these. These forms determine a basis of left invariant 1-forms $\omega^{\alpha}$, $\alpha$, and a basis of left invariant vector fields $X_{\alpha}, H_{\alpha}$. Clearly

$$
d \omega^{\alpha}=-\alpha \wedge \omega^{\alpha}-\frac{1}{2} \sum_{\beta+\gamma=\alpha} N_{\beta \gamma} \omega^{\beta} \wedge \omega^{\gamma}, \quad d \alpha=-\sum_{\beta} \frac{\langle\alpha, \beta\rangle}{\langle\beta, \beta\rangle} \omega^{\beta} \wedge \omega^{-\beta},
$$

with sums over all roots. To be more precise $\omega^{\alpha}, \alpha$ is not quite a basis of 1 -forms, since there will be relations among the $\alpha$-forms in general. To produce a basis, we would have to restrict to the $\alpha 1$-forms which are simple roots, but include all of the $\omega^{\alpha} 1$-forms, even for nonsimple $\alpha$. The basis $\omega^{\alpha}, \alpha$ is not the dual basis to $X_{\alpha}, H_{\alpha}$.

Definition 2. If $G / P$ is a rational homogeneous variety, let $\delta=\delta_{G / P}$ be

$$
\delta=\frac{1}{2} \sum_{\alpha}^{\times} \alpha
$$

where $\sum^{\times}$means the sum over all noncompact negative roots.

Lemma 2. The Killing form inner product $\langle\delta, \beta\rangle$ (where $\delta$ is half the sum of noncompact negative roots, and $\beta$ any root) vanishes just precisely for $\beta$ a root of the maximal semisimple subalgebra $\mathfrak{m} \subset \mathfrak{p}$.

Proof. Knapp [20, Corollary 5.100, p. 330] gives a completely elementary proof. We give a proof along the same lines, to keep our exposition self-contained. Pick $\beta$ any positive root. If $\gamma$ is any noncompact negative root, and $\langle\gamma, \beta\rangle>0$, then

$$
\gamma, \gamma-\beta, \gamma-2 \beta, \ldots, \gamma-q \beta=r_{\beta} \gamma
$$

is a string of roots ending in the reflection $r_{\beta}$ of $\gamma$. To start with, $\gamma$ already contains a positive multiple of a noncompact negative simple root. Equivalently, $\gamma$ has some negative multiple of a noncompact positive simple root $\alpha_{1}$. Subtracting the positive root $\beta$ can only make the multiple of $\alpha_{1}$ larger negative. Therefore the entire string consists of noncompact negative roots.

If we have an entire $\beta$-string of noncompact negative roots, for a positive root $\beta$, clearly

$$
\left\langle r_{\beta} \gamma, \beta\right\rangle=-\left\langle r_{\beta} \gamma, r_{\beta} \beta\right\rangle=-\langle\gamma, \beta\rangle .
$$

Therefore $\langle\gamma, \beta\rangle$ cancels with $\left\langle r_{\beta} \gamma, \beta\right\rangle$ in the sum $\langle\delta, \beta\rangle$. Hence the entire string cancels out of that sum.

It follows that

$$
\langle\delta, \beta\rangle=\sum_{\gamma}\langle\gamma, \beta\rangle
$$


where the sum is over noncompact negative roots $\gamma$ for which both $\langle\gamma, \beta\rangle \leq 0$ and for which the other end of the $\beta$-string through $\gamma$ is noncompact positive. Of course, the terms with $\langle\gamma, \beta\rangle=0$ cancel out too, so the sum (1) is over noncompact negative roots $\gamma$ for which both $\langle\gamma, \beta\rangle<0$ and for which the other end of the $\beta$-string through $\gamma$ is noncompact positive. In particular, the sum (1) is a sum of negative terms. But there might not be any terms.

If $\beta$ is a compact root, then clearly reflection in $\beta$ preserves the roots belonging to the parabolic subalgebra $\mathfrak{p}$, and therefore preserves the noncompact negative roots. So the noncompact negative roots will all lie in $\beta$-strings, and $\langle\delta, \beta\rangle=0$ for these roots. On the other hand, if $\beta$ is a noncompact root, then $\beta$ is either positive or negative. We can assume that $\beta$ is positive, since we only need to show that $\langle\delta, \beta\rangle \neq 0$. Take $\gamma=-\beta$, to see that the sum (1) has at least one negative term.

\section{$5 \quad$ Parabolic geometries}

The standard reference on parabolic geometries is Čap and Slovak [7]; we will use the standard definitions, as in their book, which are far too long to put into this paper. Suppose that $E \rightarrow M$ is a holomorphic parabolic geometry, with some model $G / P$. Very similar structure equations hold for any holomorphic parabolic geometry with the same model. Indeed, the Cartan connection is a 1-form valued in the Lie algebra $\mathfrak{g}$ of $G$, so splits into a sum of 1-forms $\omega^{\alpha}$ and $\alpha$ from the decomposition of $\mathfrak{g}$ into root spaces. From the definition of a Cartan geometry, the Cartan connection satisfies the same structure equations as the Maurer-Cartan form on the model, but with semibasic curvature correction terms, so

$$
\begin{aligned}
& d \omega^{\alpha}=-\alpha \wedge \omega^{\alpha}-\frac{1}{2} \sum_{\beta+\gamma=\alpha} N_{\beta \gamma} \omega^{\beta} \wedge \omega^{\gamma}+\sum_{\beta, \gamma}^{\times} \kappa_{\beta \gamma}^{\alpha} \omega^{\beta} \wedge \omega^{\gamma}, \\
& d \alpha=-\sum_{\beta} \frac{\langle\alpha, \beta\rangle}{\langle\beta, \beta\rangle} \omega^{\beta} \wedge \omega^{-\beta}+\sum_{\beta, \gamma}^{\times} \lambda_{\beta \gamma}^{\alpha} \omega^{\beta} \wedge \omega^{\gamma},
\end{aligned}
$$

where the $\kappa$ and $\lambda$ terms are Cartan geometry curvature terms, so they vanish except possibly for $\beta$ and $\gamma$ noncompact negative roots, and once again $\sum^{x}$ means the sum over noncompact negative roots.

It is vital in the following that, even if we work on a manifold where we have imposed some relations on these 1 -forms, we will still use the Killing form on the original Lie algebra $\mathfrak{g}$ to compute inner products $\langle\alpha, \beta\rangle$. This is our only notational ambiguity.

\section{Proofs of the theorems}

Replacing our Calabi-Yau manifold by a finite covering space if needed, we can assume that it bears a nowhere-vanishing holomorphic volume form. We then derive our theorem from the following stronger theorem:

Theorem 2. If a complex manifold bears a holomorphic parabolic geometry and a holomorphic volume form, then it admits a canonical holomorphic reduction of the structure group of the parabolic geometry to a reductive algebraic group.

Proof. Suppose that $E \rightarrow M$ is a holomorphic parabolic geometry modelled on $G / P$, and $\sigma$ a holomorphic volume form on $M$. Pick a Chevalley basis. Let

$$
\Omega=\bigwedge_{\alpha}^{\times} \omega^{\alpha}, \quad \delta=\frac{1}{2} \sum_{\alpha}^{\times} \alpha,
$$


where the wedge product and sum are over noncompact negative roots. The sign of $\Omega$ depends on a choice of ordering of the noncompact negative roots, but any ordering can be chosen, as long as we are consistent.

We claim that $d \Omega=-2 \delta \wedge \Omega$. Order the noncompact negative roots arbitrarily as $\alpha_{1}, \alpha_{2}, \ldots$. Expand out $d \Omega$ :

$$
d \Omega=\sum_{j}(-1)^{j+1} \bigwedge_{i<j} \omega^{\alpha_{i}} \wedge d \omega^{\alpha_{j}} \wedge \bigwedge_{i>j} \omega^{\alpha_{i}}
$$

by passing the exterior derivative operator along the various factors, hitting one $\omega^{\alpha}$ at a time, and sticking a suitable \pm sign in front. Plug in the equation for $d \omega^{\alpha}$, and the curvature terms all vanish because they occur in pairs of $\omega^{\beta} \wedge \omega^{\gamma}$, and at least one of these $\omega^{\beta}$ or $\omega^{\gamma}$ is still present in factors in that term. If we find a term like $N_{\beta \gamma} \omega^{\beta} \wedge \omega^{\gamma}$ in $d \omega^{\alpha}$, then we must have $\alpha=\beta+\gamma$. Since $\alpha$ is a noncompact negative root, at least one of $\beta$ and $\gamma$ must be as well. Neither can be equal to $\alpha$, since $N_{0 \alpha}=0$. Therefore each such term drops out of $d \Omega$. The reader now only has to check the signs to see that $d \Omega=-2 \delta \wedge \Omega$.

Our nonzero section $\sigma$ of the canonical bundle of $M$ can be pulled back to $E$ as $\sigma=s \Omega$, for a unique nowhere-vanishing function $s: E \rightarrow \mathbb{C}$. If $\sigma$ is holomorphic, then

$$
0=d \sigma=d s \wedge \Omega+s d \Omega=(d s-2 s \delta) \wedge \Omega .
$$

Let $P_{0} \subset P$ be the subgroup of $P$ acting trivially on $\Lambda^{\text {top, } 0}(\mathfrak{g} / \mathfrak{p})$. Let $E^{\prime} \subset E$ be the set of points at which $s=1$. Then $E^{\prime} \subset E$ is a smooth hypersurface since $d s \neq 0$ on tangent spaces of $E$ along $E^{\prime}$. Clearly $E^{\prime}$ is a principal right $P_{0}$-bundle.

On $E^{\prime}, \delta \wedge \Omega=0$. Therefore $\delta$ is semibasic on $E^{\prime}$ :

$$
\delta=\sum_{\alpha}^{\times} t_{\alpha} \omega^{\alpha},
$$

a sum over noncompact negative roots $\alpha$, for some functions $t_{\alpha}: E^{\prime} \rightarrow \mathbb{C}$. Taking exterior derivative, we find

$$
\begin{aligned}
0= & d\left(\delta-\sum_{\alpha} t_{\alpha} \omega^{\alpha}\right)=\sum_{\alpha}\left(d \alpha-d t_{\alpha} \wedge \omega^{\alpha}-t_{\alpha} d \omega^{\alpha}\right) \\
= & -\sum_{\beta} \frac{\langle\delta, \beta\rangle}{\langle\beta, \beta\rangle} \omega^{\beta} \wedge \omega^{-\beta}-\sum_{\alpha}\left(d t_{\alpha}-t_{\alpha} \alpha\right) \wedge \omega^{\alpha} \\
& -\frac{1}{2} \sum_{\alpha} t_{\alpha} \sum_{\beta+\gamma=\alpha} N_{\beta \gamma} \omega^{\beta} \wedge \omega^{\gamma} \quad(\bmod \text { semibasic terms }) .
\end{aligned}
$$

In particular, for any noncompact negative root $\alpha$,

$$
\mathcal{L}_{X_{-\alpha}} t_{\alpha}=2 \frac{\langle\delta, \alpha\rangle}{\langle\alpha, \alpha\rangle} .
$$

Since $-\alpha$ is a positive root, the corresponding root vector lies in $\mathfrak{p}$. Moreover, this root vector lies in the nilpotent radical of $\mathfrak{p}$, since it is a positive root. The nilpotent radical acts trivially on $\Lambda^{\text {top,0 }}(\mathfrak{g} / \mathfrak{p})$, as nilpotent groups have no nontrivial 1-dimensional representations. Every vector in $\mathfrak{p}$ gives rise to a vector field giving the associated infinitesimal action, and for the root vector of $-\alpha$, this vector field is $X_{-\alpha}$, by definition of a Cartan geometry. Since the root vector lies in the Lie algebra of $P_{0}$, the vector field $X_{-\alpha}$ generates a 1-parameter subgroup of $P_{0}$. The vector field $X_{-\alpha}$ is therefore tangent to the fibers of $E^{\prime} \rightarrow M$. 
On the fibers the vector field $X_{-\alpha}$ is a left invariant vector field. Therefore $X_{-\alpha}$ is complete. Starting at any point of $E^{\prime}$, we can move in the direction $X_{-\alpha}$ of the nilpotent part of the structure group, altering the value of $t_{\alpha}$ at a constant rate until it reaches 0 . Indeed $t_{\alpha}$ is acted on by the nilradical of the structure group as translations in the left action on $E^{\prime}$. The set of points $E^{\prime \prime} \subset E^{\prime}$ on which all $t_{\alpha}$ vanish is a smooth embedded submanifold, because its tangent space is cut out by equations

$$
\frac{\langle\delta, \alpha\rangle}{\langle\alpha, \alpha\rangle} \omega^{-\alpha}=\text { semibasic }
$$

for all noncompact negative roots $\alpha$. The structure group is reduced to a reductive algebraic group, since we have eliminated the nilradical of the original structure group, leaving only the root spaces $\alpha$ for which neither $\alpha$ nor $-\alpha$ is noncompact negative, i.e. the root spaces of the maximal reductive subgroup $M A$ of the structure group $P$. We have also eliminated the part of $A$ which acts nontrivially on the holomorphic volume forms, so our structure group is now $M A^{0}$, with $A^{0}$ the subgroup of $A$ fixing a volume form on $\mathfrak{g} / \mathfrak{p}$.

Remark 1. On a complex manifold with a meromorphic section of the canonical bundle, it would be interesting to consider what happens to this argument as we approach the zeroes or poles of the meromorphic section.

Corollary 1. If a complex manifold bears a holomorphic parabolic geometry and a holomorphic volume form, then it admits a canonical holomorphic Weyl structure.

Proof. Suppose that $E \rightarrow M$ is a holomorphic parabolic geometry modelled on $G / P$. Write the Langlands decomposition of $P$ as $P=M A N$. Let $G^{0}=P / N$. Recall that a holomorphic Weyl structure is a $G^{0}$-equivariant holomorphic section of the bundle $E \rightarrow E / N$ [7]. By Theorem 2, we have a principal right $M A^{0}$-subbundle $E^{\prime \prime} \subset E$, which induces a principal right $M A$-subbundle $E^{\prime \prime} \times_{M A^{0}} M A \subset E$. Clearly $P / N=M A$ and the map $E_{0} \rightarrow E / N$ is an isomorphism of $M A$-bundles.

\section{Parabolic geometries on tori}

Example 2. Suppose that $L$ is a Lie group with Lie algebra $\mathfrak{l}$, and write the left invariant Maurer-Cartan 1 -form on $L$ as $\ell^{-1} d \ell$. Suppose that $G$ is a Lie group and $H \subset G$ is a closed subgroup. Take any linear injection $t: \mathfrak{l} \rightarrow \mathfrak{g}$ so that the image is complementary to $\mathfrak{h}$. Then let $M=L$ and $E=M \times H$. Let $\omega \in \Omega^{1}(E) \otimes \mathfrak{g}$ be the 1 -form

$$
\omega=h^{-1} d h+\operatorname{Ad}(h)^{-1}\left(t \ell^{-1} d \ell\right) .
$$

It is easy to check that $\omega$ is the Cartan connection of Cartan geometry on $L$ modelled on $G / H$. The group $L$ acts as Cartan geometry automorphisms by the obvious action. The curvature is

$$
d \omega+\frac{1}{2}[\omega, \omega]=\frac{1}{2} \operatorname{Ad}(h)^{-1}\left(\left[t \ell^{-1} d \ell, t \ell^{-1} d \ell\right]-t\left[\ell^{-1} d \ell, \ell^{-1} d \ell\right]\right) .
$$

In particular, the Cartan geometry is flat if and only if $t$ is a Lie algebra homomorphism. See [13] for details. Clearly the group $\operatorname{Aut}(L) \times \operatorname{Aut}(G, H)$ acts as isomorphisms of these geometries.

Lemma 3. Every left invariant Cartan geometry on a Lie group is equivariantly isomorphic to one constructed as in Example 2. 
Proof. Take any Cartan geometry $E \rightarrow L$ on a Lie group $L$. Suppose that $L$ acts on $E$ lifting its left action on itself, commuting with the $H$-action, and preserving the Cartan connection. Pick any point $e_{0} \in E$. Map

$$
(\ell, h) \in L \times H \mapsto \ell e_{0} h .
$$

Clearly this is an isomorphism of principal bundles, so from now on we take $E=L \times H$. Unwinding the definition of a Cartan connection immediately yields that every Cartan connection on the bundle $L \times H \rightarrow L$ has the form

$$
\omega=h^{-1} d h+\operatorname{Ad}(h)^{-1}(\gamma)
$$

where $\gamma$ is a 1 -form on $L$ valued in $\mathfrak{g}$, so that $\gamma+\mathfrak{h}$ is a linear isomorphism, i.e. $\gamma_{\ell}: T_{\ell} L \rightarrow \mathfrak{g}$ is a linear injection, for each $\ell \in L$, and $T_{\ell} L \rightarrow \mathfrak{g} \rightarrow \mathfrak{g} / \mathfrak{p}$ is a linear isomorphism. By translation invariance

$$
\gamma=t \ell^{-1} d \ell
$$

for a unique linear map $t$ and $t \rightarrow \mathfrak{g} \rightarrow \mathfrak{g} / \mathfrak{p}$ is a linear isomorphism.

Theorem 3. Every holomorphic parabolic geometry on any complex torus is translation invariant, and obtained by the construction of Example 2 (where the Lie group $L$ is the complex torus itself). More generally, if $M$ is any compact complex manifold with holomorphically trivial tangent bundle, then $M=L / \Gamma$ for some discrete subgroup $\Gamma \subset L$ of a complex Lie group $L$. Every holomorphic parabolic geometry on $M$ is obtained by the construction of Example 2 applied to $L$, and then quotiented by $\Gamma$.

Proof. If $M$ is a compact complex manifold with holomorphically trivial tangent bundle, then any basis of the holomorphic tangent space $T_{m} M$ at any point $m \in M$ extends to a framing by holomorphic vector fields, say $X_{1}, X_{2}, \ldots, X_{n}$. These then generate a transitive complex Lie group action, say of a complex Lie group $L$. Brackets of these holomorphic vector fields can be rewritten in terms of the vector fields themselves

$$
\left[X_{i}, X_{j}\right]=\sum c_{i j}^{k} X_{k}
$$

since the $X_{k}$ form a basis. The holomorphic functions $c_{i j}^{k}$ are constant because $M$ is compact. Therefore $L$ has the same dimension as $M$, and so $M=L / \Gamma$ [33].

Following the proof of Theorem 2, the structure group of any holomorphic parabolic geometry $E \rightarrow M$ reduces to a reductive group, $G^{\prime \prime}$ on some subbundle $E^{\prime \prime} \subset E$. The Cartan connection $\omega$ splits into a sum corresponding to the splitting of $\mathfrak{g}$ into $G^{\prime \prime}$-invariant subspaces, and $\omega^{\prime \prime}$ (the part valued in $\left.\mathfrak{g}^{\prime \prime}\right)$ is a connection form for $E^{\prime \prime} \rightarrow M$. Take a global holomorphic coframing on $M$, i.e. a set of linearly independent 1 -forms $\xi^{\alpha}$ forming a basis of each cotangent space of $M$, for $\alpha$ varying over noncompact negative roots. Define a map $e \in E^{\prime \prime} \rightarrow h \in \mathrm{GL}(\mathfrak{g} / \mathfrak{p})$, by $\omega^{\alpha}=h_{\beta}^{\alpha} \xi^{\beta}$ (for $\alpha$ and $\beta$ varying over noncompact negative roots), and $h(e)=\left(h_{\beta}^{\alpha}\right)$ in the basis $X_{\alpha}$ for the sum of noncompact negative root spaces. Under right $G^{\prime \prime}$-action,

$$
h\left(r_{g} e\right)=g^{-1} h(e)
$$

for $g \in G^{\prime \prime}$. Therefore the quotient map $E \rightarrow \mathrm{GL}(\mathfrak{g} / \mathfrak{p}) / G^{\prime \prime}$ descends to a map $M \rightarrow \mathrm{GL}(\mathfrak{g} / \mathfrak{p}) / G^{\prime \prime}$. The quotient GL $(\mathfrak{g} / \mathfrak{p}) / G^{\prime \prime}$ is an affine variety: see Mumford et al. [29, Theorem 1.1, p. 27] and Procesi [30, Theorem 2, p. 556]. Affine coordinate functions will pull back to functions on $M$, and therefore must be constant. Therefore the map $M \rightarrow \mathrm{GL}(\mathfrak{g} / \mathfrak{p}) / G^{\prime \prime}$ is constant. We have an isomorphism

$$
e \in E^{\prime \prime} \rightarrow(\pi(e), h(e)) \in M \times G^{\prime \prime}
$$


trivializing the bundle $E^{\prime \prime}$. We can therefore assume that $E^{\prime \prime}=M \times G^{\prime \prime}$ and $E=M \times H$. Again unwinding the definition of a Cartan geometry,

$$
\omega=h^{-1} d h+\operatorname{Ad}(h)^{-1} \gamma,
$$

and $\gamma \in \Omega^{1}(M) \otimes \mathfrak{g}$. The functions $\left.X_{i}\right\lrcorner \gamma$ are holomorphic functions on $M$, so constant, so $\gamma$ pulls back to $L$ to be $\gamma=t \ell^{-1} d \ell$ for some constant linear map $t: \mathfrak{l} \rightarrow \mathfrak{g}$.

Remark 2. This theorem is more remarkable if one remembers that there are holomorphic Cartan geometries on complex tori which are not translation invariant.

Remark 3. Suppose that $G$ is a complex semisimple Lie group and $P \subset G$ is a parabolic subgroup. If $\mathfrak{g}$ has no Abelian subalgebra complementary to $\mathfrak{p}$, then there is no flat holomorphic $G / P$-geometry on any complex torus. Indeed this occurs for all $G / P$ which are not compact Hermitian symmetric spaces. More generally, for any $G$ and $P$, there is some complex linear subspace in $\mathfrak{g}$ complementary to $\mathfrak{p}$ which is not an Abelian subalgebra. Therefore there is a holomorphic $G / P$-geometry on any complex torus of the appropriate dimension which is not flat.

Corollary 2. If a compact Kähler manifold with $c_{1}=0$ bears a parabolic geometry, then it is covered by a torus, and the parabolic geometry pulls back to a translation invariant parabolic geometry on the torus.

Definition 3. Suppose that $P_{-} \subset P_{+}$are two closed subgroups of a Lie group $G$, so that we have a fiber bundle map $G / P_{-} \rightarrow G / P_{+}$. Let $E \rightarrow M$ be a Cartan geometry modelled on $G / P_{+}$. Then $E \rightarrow E / P_{-}$is a Cartan geometry modelled on $G / P_{-}$, called the lift of the Cartan geometry on $M$.

Corollary 3. On any compact homogeneous Kähler manifold, all parabolic geometries are lifted (as in Definition 3) from a translation invariant geometry on a torus (constructed as in Example 2). In particular, all such parabolic geometries are homogeneous.

Proof. Borel and Remmert [6] proved that every compact homogeneous Kähler manifold is a product of a torus and a rational homogeneous variety. The rational homogeneous variety bears rational curves just when it has positive dimension. These rational curves ensure that the parabolic geometry is lifted from lower dimension (see Biswas and McKay [4]), quotienting out the rational homogeneous variety entirely.

Remark 4. Any parabolic geometry on any rational homogeneous variety (or more generally, on any compact rationally connected complex manifold) is flat and isomorphic to its model (see Biswas and McKay [4]).

\section{Conclusion}

The classification of holomorphic parabolic geometries on compact complex manifolds with $c_{1}>0$ is complete [4] (and more generally the classification on rationally connected compact complex manifolds). Above we give the classification of holomorphic parabolic geometries on compact Kähler manifolds with $c_{1}=0$. The classification for $c_{1}<0$ must be more difficult, as there are many locally symmetric varieties and many holomorphic projective connections on compact Riemann surfaces of genus $g \geq 2$.

We propose a conjecture: if $M$ is a compact complex manifold with $c_{1}<0$, then either (1) $M$ admits no parabolic geometry, or (2) $M$ admits a parabolic geometry modelled on a compact Hermitian symmetric space $G / P$ and $M$ is covered by the noncompact dual of that symmetric space. In case (2), every parabolic geometry on $M$ modelled on $G / P$ is flat, and if the 
factorization of $G / P$ into a product of irreducible compact Hermitian symmetric spaces has no factor of dimension 1 (i.e. isomorphic to $\mathbb{P}^{1}$ ) then the parabolic geometry on $M$ pulls back to the standard flat parabolic geometry on the noncompact dual.

The above conjecture was proven by Kobayashi [21] with the additional hypothesis that the parabolic geometry is a normal conformal connection or a normal projective connection.

There are examples of smooth complex projective varieties which are not locally symmetric and which have holomorphic projective connections [16]. These examples stand in the way of any obvious conjecture as to which smooth complex projective varieties have holomorphic parabolic geometries. They have $c_{1} \leq 0$ but not $c_{1}<0$ or $c_{1}=0$. It might help to be able to limit the possible models as follows.

We propose another conjecture: suppose that $M$ is a compact connected Kähler manifold, bearing a holomorphic parabolic geometry. Then the canonical bundle of $M$ is not pseudoeffective if and only if the parabolic geometry drops (in the sense of [4]) to a holomorphic parabolic geometry on a lower dimensional compact Kähler manifold. (We can essentially ignore such parabolic geometries in any classification.) In the other direction, if the canonical bundle of $M$ is pseudoeffective, and the parabolic geometry is regular at at least one point, then the model is a compact Hermitian symmetric space. (Consequently we hope to reduce the classification to the classification of holomorphic parabolic geometries modelled on compact Hermitian symmetric spaces, a topic which presumably lies close to the study of locally Hermitian symmetric varieties.)

We propose another conjecture, concerning the complex torus: on any complex torus, a holomorphic Cartan geometry is translation invariant if and only if it is locally homogeneous.

We expect that foliations play a fundamental role in the phenomenon of translation invariance. We conjecture: suppose that $G$ is a complex Lie group and $H \subset G$ is a closed complex subgroup. Then either (1) every $G$-invariant holomorphic foliation of $G / H$ has a $G$-invariant holomorphic complementary subbundle of the tangent bundle and every holomorphic Cartan geometry modelled on $G / H$ on any complex torus is translation invariant or (2) there is a $G$ invariant holomorphic foliation of $G / H$, with no invariant holomorphic complementary subbundle of the tangent bundle, and there is a complex Abelian variety $A$ and a holomorphic Cartan geometry on $A$ modelled on $G / H$ which is not translation invariant.

\section{Acknowledgements}

This material is based upon works supported by the Science Foundation Ireland under Grant No. MATF634.

\section{References}

[1] Atiyah M.F., Complex analytic connections in fibre bundles, Trans. Amer. Math. Soc. 85 (1957), $181-207$.

[2] Berger M., Lascoux A., Variétés Kähleriennes compactes, Lecture Notes in Mathematics, Vol. 154, SpringerVerlag, Berlin, 1970.

[3] Biswas I., McKay B., Holomorphic Cartan geometries and Calabi-Yau manifolds, J. Geom. Phys. 60 (2010), 661-663, arXiv:0812.3978.

[4] Biswas I., McKay B., Holomorphic Cartan geometries and rational curves, arXiv:1005.1472.

[5] Biswas I., McKay B., Holomorphic Cartan geometries, Calabi-Yau manifolds and rational curves, Differential Geom. Appl. 28 (2010), 102-106, arXiv:1009.5801.

[6] Borel A., Remmert R., Über kompakte homogene Kählersche Mannigfaltigkeiten, Math. Ann. 145 (1961/1962), 429-439.

[7] Čap A., Slovák J., Parabolic geometries. I. Background and general theory, Mathematical Surveys and Monographs, Vol. 154, American Mathematical Society, Providence, RI, 2009. 
[8] Dumitrescu S., Structures géométriques holomorphes sur les variétés complexes compactes, Ann. Sci. École Norm. Sup. (4) 34 (2001), 557-571.

[9] Dumitrescu S., Connexions affines et projectives sur les surfaces complexes compactes, Math. Z. 264 (2010), 301-316, arXiv:0805.2816.

[10] Dumitrescu S., Killing fields of holomorphic Cartan geometries, Monatsh. Math. 161 (2010), 145-154, arXiv:0902.2193.

[11] Fulton W., Harris J., Representation theory. A first course, Graduate Texts in Mathematics, Vol. 129, Springer-Verlag, New York, 1991.

[12] Gunning R.C., On uniformization of complex manifolds: the role of connections, Mathematical Notes, Vol. 22, Princeton University Press, Princeton, N.J., 1978.

[13] Hammerl M., Homogeneous Cartan geometries, Arch. Math. (Brno) 43 (2007), 431-442, math.DG/0703627.

[14] Igusa J.-I., On the structure of a certain class of Kaehler varieties, Amer. J. Math. 76 (1954), 669-678.

[15] Inoue M., Kobayashi S., Ochiai T., Holomorphic affine connections on compact complex surfaces, J. Fac. Sci. Univ. Tokyo Sect. IA Math. 27 (1980), 247-264.

[16] Jahnke P., Radloff I., Threefolds with holomorphic normal projective connections, Math. Ann. 329 (2004), 379-400, math.AG/0210117.

[17] Jahnke P., Radloff I., Projective threefolds with holomorphic conformal structure, Internat. J. Math. 16 (2005), 595-607, math.AG/0406113.

[18] Klingler B., Structures affines et projectives sur les surfaces complexes, Ann. Inst. Fourier (Grenoble) 48 (1998), 441-477.

[19] Klingler B., Un théorème de rigidité non-métrique pour les variétés localement symétriques hermitiennes, Comment. Math. Helv. 76 (2001), 200-217.

[20] Knapp A.W., Lie groups beyond an introduction, 2nd ed., Progress in Mathematics, Vol. 140, Birkhäuser Boston Inc., Boston, MA, 2002.

[21] Kobayashi S., Horst C., Topics in complex differential geometry, in Complex Differential Geometry, $D M V$ Sem., Vol. 3, Birkhäuser, Basel, 1983, 4-66.

[22] Kobayashi S., Ochiai T., Holomorphic projective structures on compact complex surfaces, Math. Ann. 249 (1980), 75-94.

[23] Kobayashi S., Ochiai T., Holomorphic projective structures on compact complex surfaces. II, Math. Ann. 255 (1981), 519-521.

[24] Kobayashi S., Ochiai T., Holomorphic structures modeled after compact Hermitian symmetric spaces, in Manifolds and Lie Groups (Notre Dame, Ind., 1980), Progr. Math., Vol. 14, Birkhäuser, Boston, Mass., 1981, 207-222.

[25] Kobayashi S., Ochiai T., Holomorphic structures modeled after hyperquadrics, Tôhoku Math. J. (2) 34 (1982), 587-629.

[26] McKay B., Characteristic forms of complex Cartan geometries, Adv. Geom. 11 (2011), 139-168, arXiv:0704.2555.

[27] McKay B., Holomorphic Cartan geometries on uniruled surfaces, C. R. Acad. Sci. Paris 349 (2011), 893-896, arXiv:1105.4732.

[28] McKay B., Pokrovskiy A., Locally homogeneous geometric structures on Hopf surfaces, Indiana Univ. Math. J. 59 (2010), 1491-1540, arXiv:0910.0369.

[29] Mumford D., Fogarty J., Kirwan F., Geometric invariant theory, 3rd ed., Ergebnisse der Mathematik und ihrer Grenzgebiete (2), Vol. 34, Springer-Verlag, Berlin, 1994.

[30] Procesi C., Lie groups. An approach through invariants and representations, Universitext, Springer, New York, 2007.

[31] Serre J.-P., Complex semisimple Lie algebras, Springer Monographs in Mathematics, Springer-Verlag, Berlin, 2001.

[32] Sharpe R.W., Differential geometry. Cartan's generalization of Klein's Erlangen program, Graduate Texts in Mathematics, Vol. 166, Springer-Verlag, New York, 1997.

[33] Wang H.-C., Closed manifolds with homogeneous complex structure, Amer. J. Math. 76 (1954), 1-32.

[34] Yau S.T., Calabi's conjecture and some new results in algebraic geometry, Proc. Nat. Acad. Sci. U.S.A. 74 (1977), 1798-1799. 